\title{
TRASCENDENCIA DEL PRIMER SAGGIO SULLA STORIA NATURALE DEL CHILI DE J.I. MOLINA, SU TRADUCCION, EL COMPENDIO ANÓNIMO Y EL BICENTENARIO
} José R. STUARDO*

\section{RESUMEN}

Hay una única traducción al español de la obra del abate J.I. Molina Saggio sulla storia naturale del Chili, publicada originalmente en 1782, que fue hecha en 1788 bajo el nombre equivocado de Compendio, lo que dio origen a una seguidilla de interpretaciones que han oscurecido la trascendencia de aportes anteriores de otros autores que permitieron esta publicación. Se analiza la importancia de esta obra desde el punto de vista de las primeras especies animales chilenas descritas, usando la nomenclatura binomial propuesta por el gran naturalista Carlos Linneo (o de Linneo). Fue el uso de nombres génericos y específicos en latín, lo que permitió la incorporación de un número importante de los nombres propuestos por Molina a la literatura de la taxonomía zoológica internacional.

Palabras claves: Historia de la ciencia, zoología, Chile, J.I. Molina, C. Linneo.

\section{ABSTRACT}

The only translation into Spanish of J.I.Molina's work Saggio sulla storia naturale del Chili originally published in 1782, was done in 1788 under the erroneous name of Compendio, an unfortunate decision which gave rise over time to a series of misinterpretations obscuring the importance of previous works by other authors which, indeed, made this publication possible. The importance of this Saggio... is analyzed from the point of view of the first Chilean animal species described using the binomial system of nomenclature proposed by the great naturalist Carolus Linnaeus. The decision to use generic and specific names in Latin allowed the incorporation of an important number of the names proposed by Molina into the international zoological taxonomic literature.

Keywords: History of Science, Zoology, Chile, J.I. Molina, C.Linnaeus.

Recibido: 18.09.2006. Aprobado: 11.04.2007.

* Profesor del Depto. de Oceanografía, Facultad de Ciencias Naturales y Oceanográficas, Universidad de Concepción.Concepción, Chile. E-mail: jstuardo@udec.cl 

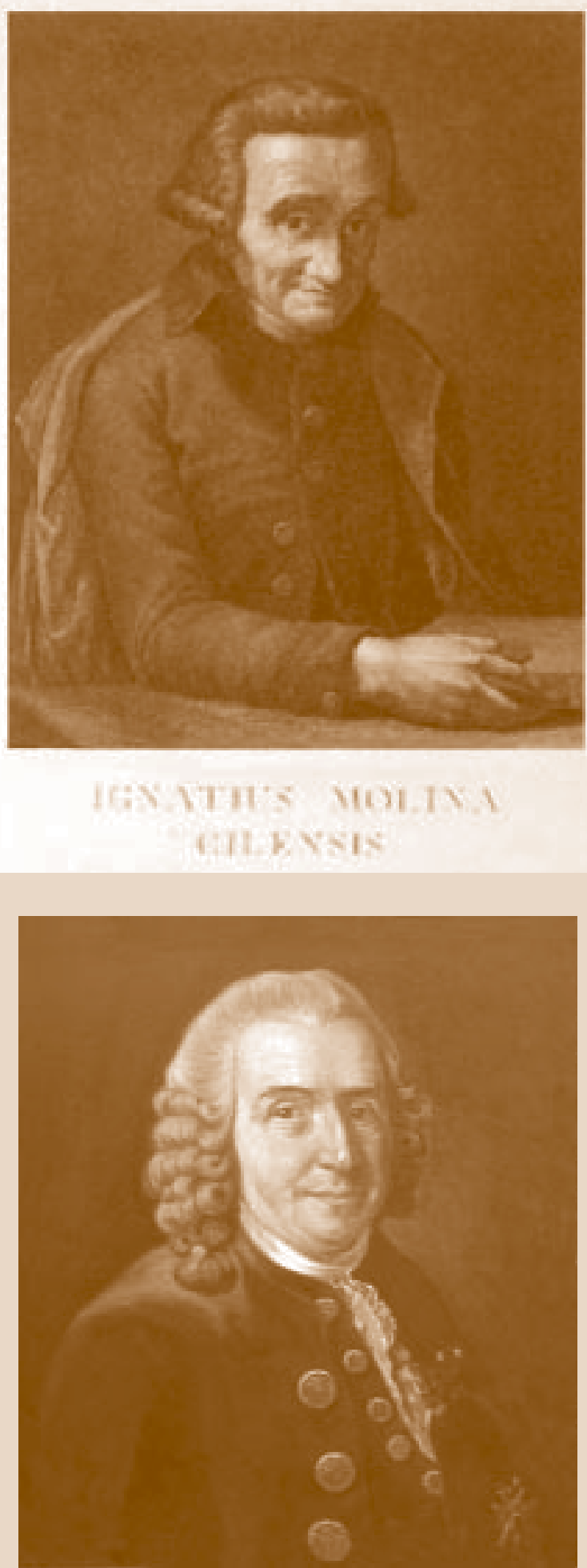

[2] Carlos Linneo. Oleo. 
$\mathrm{E}$ L ABATE Molina no es el primer autor nacional que describió plantas y animales chilenos reconocibles, pero es el primero que lo hizo usando nombres científicos, en su primera obra Saggio sulla storia naturale del Chili ${ }^{[3]}$ publicada el año 1782 en Bolonia, lo que proyectaría ya en el siglo XVIII su nombre y el de las primeras especies chilenas descritas por él a la literatura botánica y zoológica internacional y mundial. Esto ocurría en el momento en que Occidente se abría expectante al nuevo conocimiento de la geografía y la historia natural del resto del mundo, con los resultados de las observaciones y descubrimientos realizados por los numerosos viajes europeos con intereses geopolíticos, comerciales y científicos que se realizaban a Asia, América y al Mar del Sur. Se justifica identificarlo, además, con los primeros clérigos-naturalistas nacionales que impactaron a Europa con información sobre la geografía y la vida social y política de nuestro país, pero también, sobre los animales y plantas que lo caracterizan. Sin embargo, los aportes históricos de Molina respecto de la vida social y política de Chile contenidos en su segunda obra, Saggio sulla storia civile del Chile, publicada en el año 1787, son sólo complementarios a lo publicado en su época por otros autores y fueron menores comparados a la trascendencia de la información que entregó sobre la flora y fauna.

Nos referimos en este artículo sólo a su contribución sobre los animales, cuyas especies son descritas por primera vez siguiendo el denominado Sistema o Nomenclatura Binomial, usando nombres genéricos y específicos en latín junto a una diagnosis en esta lengua, como propusiera Carlos Linneo (Carolus Linnaeus o Linnaei), en su Systema Naturae en el año $1735^{[4]}$, obra tan relevante para las ciencias naturales, que se continuó en ediciones mejoradas durante las seis décadas siguientes a su primera aparición (Anexo 1). No podía ser de otra manera, dado el enorme progreso de las ciencias naturales durante el siglo XVIII, el llamado Siglo de las Luces.

Es claro que Molina, quien llegó a Imola, Italia, junto a los jesuitas desterrados en 1768, y a Bolonia en 1774, conoció una o más de las últimas ediciones de esta obra de Linneo con su propuesta para clasificar a las distintas clases de minerales, animales y plantas. Por ejemplo, la clasificación contenida en la edición 10, publicada en 1758 (que por acuerdo internacional posterior se consideró la base del desarrollo de la zoología sistemática) y, en particular, la de la edición 12 de 1766 de la misma, que aparecieron, respectivamente, 14 y 6 años antes que la primera obra de Molina.

La primera y única traducción del Saggio... de 1782 al español fue hecha por Domingo Joseph de Arquellada Mendoza (individuo de la Academia de Buenas Letras de Sevilla y maestrante de Ronda) con el nombre de Compendio de la historia geográfica, natural y civil del Reyno de Chile. Primera parte,

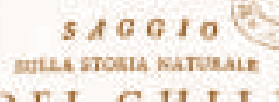

D E L C H I L I

DEL nemok ATATE

gtovassot igNazio

MOLINA.
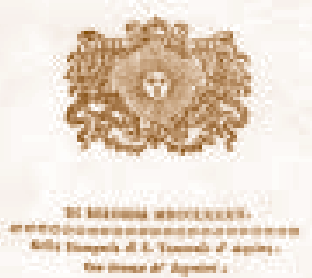

3apa del Saggio... de 1782, en italiano.

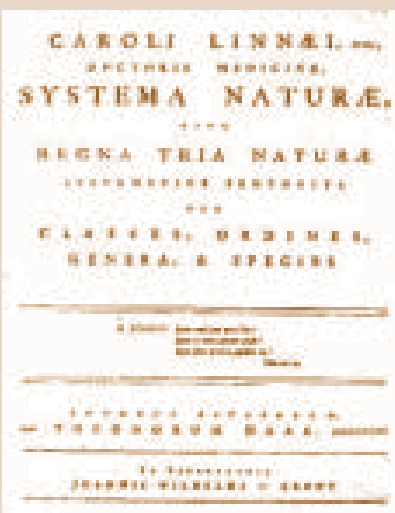

[4] Tapa del Sistema Naturae, editio 1. 
campristo

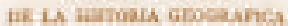

raruka $x$ civis

DEx girNo os chIE.

Ecarro Ex ratiang

goa LE ADAta DON JWAN wracin mark.

partitas pekTe.

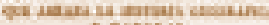

Humen merias

nos pos dos arrycs aysep

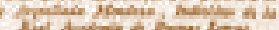

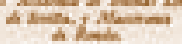

axian

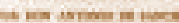

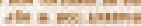

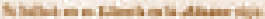

Tapa de la traduc. al español del Saggio.

\section{COMPENDIO}

Detis vroxis

stoskanes vatesask. e cievis

exis aseso

DEL CHILE.

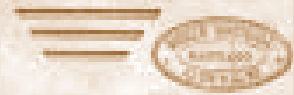

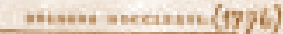

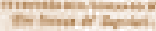

[6] Tapa del Compendio anónimo de 1776. que abraza la historia geográfica y natural ${ }^{[5]}$, publicada en Madrid el año 1788. Un facsímil de esta traducción fue impreso en el año 2000 en Santiago como parte de la Biblioteca del Bicentenario, siendo erróneamente considerado como "segunda edición" $[*]$. Efectivamente, una segunda edición de la traducción de Arquellada Mendoza ya existía publicada por José Toribio Medina en la Colección de Historiadores de Chile (1889, aunque desgraciadamente impresa con errores tipográficos), de modo que la reedición del Bicentenario es en realidad la tercera edición del mismo texto, también con errores.

Don Nicolás de la Cruz y Bahamonde tradujo al español en 1795 (1791 según Feliú Cruz, 1966: 265) el otro Saggio... de Molina sobre historia civil, ya mencionado, publicado también en Bolonia en 1787, ahora traducido con el título de Compendio de la historia civil del Reyno de Chile.

\section{LA LIBERTAD DEL TRADUCTOR}

Aquí, y sin mayor razón, Arquellada Mendoza (seguido por De la Cruz y Bahamonde) introduce el primer gran equívoco de esta historia al traducir el primer Saggio... de Molina no bajo el título de "Ensayo..." sino, paradójicamente, con el mismo nombre con el que fue publicada otra obra anterior en 1776, también en italiano pero de autor anónimo intitulada Compendio della Storia Geografica, Naturale e Civile del Regno del Chile ${ }^{[6]}$. Por su importancia, esta obra ha sido referida en la literatura nacional como "El Compendio anónimo". Así, el mismo nombre, ahora en español: “Compendio de la Historia Geografica, Natural y Civil del Reyno de Chile. Primera Parte, Que Abraza La Historia Geografica y Natural" fue impuesto por este letrado español a la primera obra de Molina.

¿Por qué tradujo la palabra Saggio por Compendio, y no tradujo el verdadero título? ¿Descriterio o mala fe? ¡Y nadie ha reclamado sobre esto! ¿Pensaba Don Domingo, al igual que algunos otros en esa época, refrendar así que la obra anónima había sido escrita también por el abate Molina? Nada dice sobre ello en su muy breve Nota del traductor (p. xix), salvo informar que esta obra había sido traducida con grandes elogios al francés y al alemán, pero sin explicar que en el primer caso la traducción de M. Gruvel al francés en 1789 fue sólo con el título que debía tener, esto es: "Essai sur l'Histoire Naturelle du Chili", y que el mismo concepto fue usado por J.D. Brandis en su traducción al alemán del mismo año: "Versuch einer Naturgeschichte von Chile" (véase Literatura citada).

En italiano existe la palabra compendio, al igual que en español y ése es, exactamente, el sentido que el autor le asigna al título de la obra anónima publicada sobre nuestro país en el año 1776, a la que ya nos hemos referido. Saggio en italiano tiene también un significado preciso en español que es el 
de ensayo. Para evitar la confusión (si no mala fe) resultante de la traducción de Arquellada Mendoza, la primera obra cuya paternidad es reconocida por Molina debió en consecuencia haberse traducido como Ensayo sobre la historia natural deChile, un título más acorde con el espíritu conque Molina lo escribió, ya que obviamente no usó el término italiano "Compendio" para diferenciarlo de esa otra obra anónima anterior. En su Prefacio dice respecto de esta y otras obras publicadas antes que la suya:

... un Compendio anónimo que se publicó en lengua italiana en 1776, y que en cierto modo nos da una noticia mas completa de Chile que la que nos han dado las demás obras impresas, singularmente en torno a la geografia, y á la Historia Natural. Mas como quiera que este Compendio es también demasiado conciso, esto mismo me ha hecho pensar que haria un obsequio util á las personas que gustan de las cosas Americanas, presentandoles otro compendio [el original en italiano no dice compendio sino "ragguaglio" que significa informe, balance] en que se traten mas á la larga y con mas individualidad las producciones y los sucesos mas notables de aquel pais (sic; nótese la tergiversación de Arquellada Mendoza en su traducción).

Es curioso como algunos autores que siguen los juicios injustificadamente repetidos de que Molina es también el autor del Compendio anónimo (lo que Molina nunca afirmó), evitan referirse a la verdadera primera obra de Molina como su primer "Ensayo..." "y algo pomposamente se refieren a ella como la Historia natural de M olina (e.g. Hanisch, 1976). Al respecto, es loable que el uso adecuado de "Ensayo" haya sido también respaldado por el Prof. Rodolfo Jaramillo, uno de los mayores biógrafos de Molina, quien lo incorpora al traducir recientemente el segundo Saggio sulla storia naturale del Chile de Molina publicado en 1810 (en el hecho la revisión del primero), como Ensayo sobre la historia natural de Chile.

\section{SIGUEN LAS LIBERTADES}

Paradójicamente, pese a todo lo publicado sobre esta primera obra de Molina, parece no haberse comentado nada sobre la estrictez de la traducción y las libertades (errores) del único traductor, incluyendo algunos pocos errores del mismo Molina, no un pecado en sí, ya que ocurre hasta hoy en día, como resultado común en la elaboración de trabajos de amplia envergadura faunística y taxonómica.

Respecto del texto, hay que reconocer que Molina escribía muy bien el italiano en un lenguaje científico claro y conciso, lo que desgraciadamente no ocurre con el español del literato Arquellada Mendoza. Por ejemplo, el 
catratogo 1.

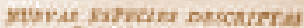

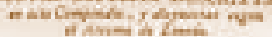

kHOKUa ANimate

Mamaina.

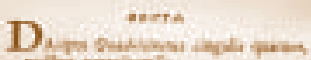

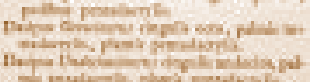

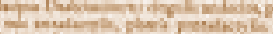

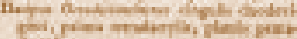

teast.

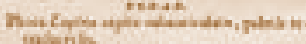

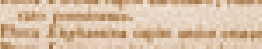

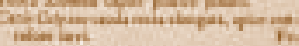

Parte inicial del Catálogo de Molina de 1782. primer párrafo del Libro IV en italiano que inicia la descripción de los animales contiene con elegante claridad 5 ideas diferentes, cada una separada por punto seguido, pero desgraciadamente traducidas al español de una tirada, sólo separadas por dos punto y coma y algunas conjunciones. Esto es característico de todo el texto.

Hay otros errores tipográficos menores en el texto traducido, pero lo que es muy grave, en la lista o Catálogo final ${ }^{[7]}$ que es el resumen técnico de todas las especies animales descritas por Molina, el traductor cambió en algunos casos y eliminó en otros por descuido, no sólo letras de palabras del texto en latín, sino que omitió también el nombre y la descripción de las dos especies siguientes:

Página 391 (de la traducción):

ANSERES (Anseres en la página 344 del texto original en italiano, después de "Anas Regia")

Anas Coscoroba rostro extremo dilata-

to, rotundato, corpore albo.

Páginas 396-397 (de la traducción):

TESTACEA (Testacea en la página 348 del texto original en italiano, después de "Chama Thaca")

M ytilus Albus testa transverse striata, natibus gib-

bis, cardine laterali.

Obviamente, estas dos especies tampoco figuran en el facsímil publicado por la Biblioteca del Bicentenario, induciendo a error a los que usen esta traducción, creyendo consultar así la traducción fiel del libro original de Molina en italiano.

Algunos de los muchos errores de escritura de palabras en latín son, por ejemplo:

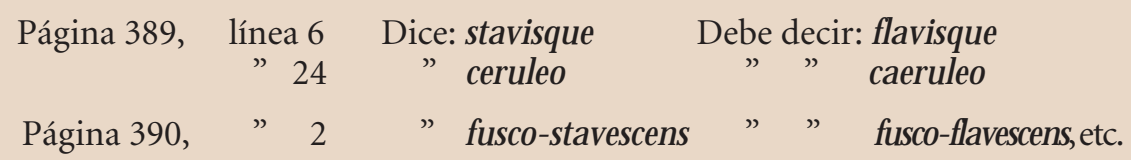

Pero, un error propio de Molina fue omitir al final de su obra, en su Catálogo I, al Orden DIPTERA en el que, de acuerdo a la clasificación de Linneo que seguía, debió incluir a su especie Tipula moschifera, que aparece como HIMENOPTERA. Un lapsus importante en la ubicación de esta especie respecto de la clasificación propuesta.

La mala traducción de algunos nombres comunes para los naturalistas, tampoco ayudó a la proyección del valor educativo de esta obra; es más, creó confusión, aunque Molina usó tales nombres en su sentido zoológico preciso. Un caso en cuestión es el término "mitoli" que se traduce como 
"almeja" (!) en lugar de mitilos o mejillones, o en último caso "choros", nombre popular que menciona Ovalle para identificar a las numerosas especies de esta familia de bivalvos no sólo abundantes y diversificados en nuestro país, sino de tanta importancia en la dieta de sus habitantes desde sus primeros inicios y hasta la actualidad. Ocurre lo mismo con otras palabras; por ejemplo, al describir el Piure, Molina dice que habita en una "colmena coriácea", que de Arquellada Mendoza traduce como "colmena glutinosa" (!). Luego, menciona a las Holoturias (refiriéndose a Medusas), y dice que "las olas del mar arrojan a la playa varias especies de Oloturias"; pero el traductor intercala la palabra "frecuentemente" que da un sentido de abundancia muy diferente a la afirmación original y a la realidad. En la misma página (189 de la traducción), Molina escribe indistintamente Sepia o Seppia, que es traducida como Jibia, incluyendo al "Pulpo". Respecto a los nombres comunes usados para algunos Testáceos (los moluscos con concha), Molina menciona "ostrichae, mitoli, came, soleni, foladi, lepadi o patelle, e de buccini", nombres que se traducen como "ostras, almejas (en lugar de mejillones o mitílidos), chamas, solénidos, navajas de barbero (otro nombre para los Solénidos, en vez de foládidos), folados o patenas (sic, en vez de lepas o lepádidos y patelas!) y buccinos". En la página siguiente Molina escribe mal el género de los mejillones como Mytulus en vez de M ytilus, aunque esto ocurre también en otras obras de su época.

En esta seguidilla de errores, que debieron haber sido comparados con el texto italiano, es también lamentable que los editores de la edición 2000, posiblemente guiados por el cambio introducido por el traductor, confunden también en la Nota del editor la fecha de publicación del Saggio de Molina de 1872 con la fecha de publicación del Compendio anónimo de 1776. ¡Todo por la descuidada pero aceptada traducción del vocablo compendio en vez de ensayo!

\section{MOLINA Y SU USO DE LAS EDICIONES DEL SYSTEM A NATURAE DE LINNEO}

En el período en que Molina llegó a Europa e inició sus estudios en ciencias naturales en Bolonia, los naturalistas discutían la mejor manera de clasificar a los animales y las plantas, lo que nuestro autor comenta refiriéndose a que le hubiera gustado seguir por ejemplo a J.-P. de Tournefort (1656-1708) para estudiar a las plantas, o a M.-J. Brisson (1723-1806 ${ }^{[8]}$ en el caso de los animales. La dificultad principal consistía en cómo identificar a las especies, lo que Linneo, un botánico, resolvió proponiendo su clasificación binomial de género y especie, concepto que Molina afortunadamente siguió.

En consecuencia, el Systema N aturae de Linneo fue no sólo el tratado en que Molina estudió taxonomía zoológica (y botánica), sino que el texto guía

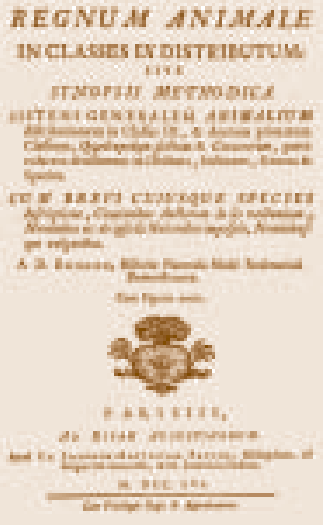

${ }^{[8]}$ Tapa de la obra de Brisson . 
CAROLI a LINNE.

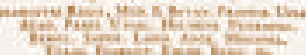

S Y S T E M A NATURA:

REGGNA TRAA NATUR,E,

clasms, aketars.

oentsa, someles.

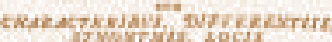

Thuis 1

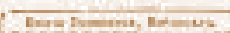

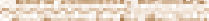

$\sin \mathrm{C}$ Mit

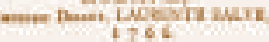

${ }^{9}$ Sistema Naturae, editio 12. para la descripción de las especies que el abate probablemente conocía y, en especial, aquellas que claramente no conocía, pero que habían sido descritas de manera general por los clérigos-naturalistas y viajeros extranjeros que ya habían publicado información sobre ellas. Molina no los menciona en el Saggio... de 1782, pero en su texto hay descripciones de especies que son transcripciones casi idénticas si no idénticas a las descripciones de estos otros autores.

Esta obra de Linneo, la base de la clasificación taxonómica seguida por el mundo científico con posterioridad, llegó a tener en su época (siglo XVIII), 13 ediciones sucesivas, todas escritas en latín. Las 12 primeras fueron publicadas por el mismo, de las cuales la inicial apareció en el año 1735 como un simple pero denso folleto de apenas 13 páginas, mientras que la edición 10, que ha sido declarada la base para la taxonomía actual de los animales, apareció en la parte correspondiente sólo a este grupo (reino animal) en un tomo de 822 páginas, impreso en el año 1758, o sea, con 35 años de diferencia, y comprendió un total de 4.370 especies (!). Esto demuestra que durante ese período el autor fue perfeccionando gradualmente su clasificación, incorporando a los nuevos órdenes y las nuevas especies descritas para el mundo conocido de su época. La edición 12, reformada ${ }^{[9]}$, incorporó lo correspondiente a los animales en el tomo 1 , con dos partes separadas publicadas en 1766 y 1767 respectivamente y un total de 1.327 páginas; ésta fue la última edición publicada por Linneo.

El tomo 1 de la edición 13, publicada por el médico y naturalista alemán Johann Friedrich Gmelin después de la muerte de Linneo, incorporó numerosas nuevas especies y géneros del reino animal incluyendo a los taxones propuestos por Molina en 1782, y tuvo seis partes, reformadas y actualizadas que aparecieron entre los años 1788 y 1793, con un total de 4.120 páginas (!). Esta última edición fue, pues, la que permitió a Molina pasar a la posteridad en la historia de la ciencia al registrarlo internacionalmente como el primer creador de especies chilenas con nombre científico y diagnosis en latín. Pero, ¿cuál de las ediciones del Systema Naturae usó Molina al publicar su primer Saggio...?

Para aclarar esto es necesario comparar la clasificación y lista de Ordenes, Géneros y Especies que Molina incluyó en su Catálogo final de especies descritas en el 1er. Saggio..., con las sucesivas clasificaciones del reino animal que aparecen en las distintas ediciones del Systema Naturae. En la Tabla 1 se presenta la lista de especies y la clasificación seguida por Molina en 1782 y en la Tabla 2, la clasificación de Ordenes y Géneros aceptados por Linneo en las ediciones 1, 10, 12 y 13 de su Systema Naturae. Como se discute a continuación, la clasificación de Molina se encuadra con los cambios introducidos por Linneo en la edición 12 de su Systema Naturae.

La clasificación de Linneo durante los 53 años en que se reeditó el Systema 
Naturae con revisiones sucesivas, mantuvo su diferenciación de 6 grandes clases animales, pero fue sufriendo modificaciones no sólo a nivel de agrupaciones mayores como son los órdenes sino que también a nivel de géneros y especies. La Tabla 2, que presenta comparativamente la evolución de los órdenes y el número de sus géneros (indicados en paréntesis después de cada uno de ellos) para cada clase, muestra una gran diferencia entre la proposición inicial (1735) y las últimas ediciones, pero con una persistencia de órdenes y géneros en casi todas las clases en las ediciones de 1758 y 1766, salvo en Mammalia y Pisces, y posteriormente en los Vermes, una clase obviamente muy artificial y por ello en ajuste permanente, como se demuestra en la edición 13. En el hecho, la falta de estudios faunísticos y el escaso número de especialistas necesarios para tales estudios, no produciría cambios importantes hasta fines del siglo XVIII, cuando las grandes expediciones europeas incorporaron activamente la recolección y descripción de especies.

De esta comparación se concluye que al escribir su primer Saggio..., Molina utilizó la clasificación propuesta en la edición de 1766, en la que el género Dasypus (el de los "quirquinchos") se incluyó en el Orden BRUTA, y nó en el orden BESTIAE anterior que desapareció, con los otros géneros ahora transferidos al Orden FERAE.

\section{ESPECIES ANIMALES DESCRITAS POR MOLINA}

Molina propuso en esta obra un total de 117 nombres específicos, de los cuales 29 eran especies de mamíferos, 34 de aves, 9 de aptera, 15 de vermes, 3 de amphibia, 11 de peces y 16 de insectos. Incluye además a dos géneros nuevos para la ciencia: Pyura y Phytotoma, correspondientes al conspicuo "piure" entre los invertebrados marinos y a la muy particular "rara" entre las aves, respectivamente, que en el tiempo dieron origen a dos nuevas familias animales: Pyuridae y Phytotomidae. De ese total sólo 53 especies son válidas en la actualidad y llevan su nombre; el resto son algunas dudosas, otras sinónimos de otras especies y otras malas, no identificables. Notable logro para un clérigo naturalista que llegó a tomar lecciones a Italia y terminó describiendo animales pertenecientes a casi todos los grupos zoológicos conocidos en su tiempo (¡y plantas!). ¿Cómo fue esto posible? ¿Conocía Molina directamente todos los animales (y plantas) que describió? Porque, ¡curiosamente, a diferencia de los autores naturalistas desu época, no dibujó a ninguno!

Aun cuando estos problemas son objeto de un extenso análisis en otra obra en elaboración, se pueden ahora discutir resumidamente. 
TABLA 1. Géneros y especies de Molina (1782), según los Ordenes de Linneo,1776. (Por error Molina no incluyó el Orden Diptera) (En marrón=especies válidas asignadas a Molina).

\begin{tabular}{|c|c|}
\hline MAMMALIA & VERMES \\
\hline BRUTA & MOLLUSCA \\
\hline $\begin{array}{l}\text { Dasypus quadricinctus; D. octocinctus; } \\
\text { D. undecimcinctus; D. octodecimcinctus }\end{array}$ & Pyura gen.nov. P. chilensis \\
\hline FERAE & Sepia unguiculata; S. tunicata; S. hexapodia \\
\hline Phoca lupina; P. porcina; P. elephantina; P. leonina & Echinus albus; E. Níger \\
\hline Canis culpaeus & TESTACEA \\
\hline Felis puma; F. gigna; F. colocola & Lepas psittacus \\
\hline Viverra chinga & Pholas chiloensis \\
\hline M ustela felina; M .cuja; M .quiqui & $\begin{array}{l}\text { Solen macha } \\
\text { Chama thaca }\end{array}$ \\
\hline GLIRES & Mytilus albus; M . ater; M. chorus \\
\hline Lepus viscacia; L. minimus & Murex locus \\
\hline Castor huidobrius & Helix serpentina \\
\hline M us cyanus; M . laniger; M . maulinus; M. coypus & AMPHIBIA \\
\hline Sciurus degus & REPTILIA \\
\hline PECORA & Rana arunco; R. lutea \\
\hline Camelus huanacus; C. vicugna; C. araucanus & Lacerta palluma \\
\hline Capra pudu & NANTES \\
\hline BELLUAE & Squalus fernandinus \\
\hline Equus bisulcus & PISCES \\
\hline AVES & APODES \\
\hline ACCIPITRES & Stromateus comarca \\
\hline Vultur jota & THORACICI \\
\hline Falco tharus & Chaetodon aureus \\
\hline Strix cunicularia & Sparus chilensis \\
\hline PICAE & ABDOMINALES \\
\hline Psittacus jaguilma; P. cyanalisios; P. choraeus & Silurusluvur \\
\hline Picuslignarius; P.pitius & Esox chilensis \\
\hline Trochilus cyanocephalus; T. galeritus; T. minimus & Mugil chilensis \\
\hline ANSERES & Cyprinus regius; C. caucus; C.malchus; C. tulus \\
\hline $\begin{array}{l}\text { Anas coscoroba; A. melancorypha; } \\
\text { A. hybrida; A. regia }\end{array}$ & INSECTA \\
\hline Diomedea chilensis; D.chiloensis & COLEOPTERA \\
\hline Pelecanus thagus & Lucanus pilmus \\
\hline GRALLAE & Chrysomela maulica \\
\hline Phaenicopterus chilensis & \\
\hline $\begin{array}{l}\text { Ardea erytrocephala; A. galatea; A. cyanocephala; } \\
\text { A. thula }\end{array}$ & LEPIDOPTERA \\
\hline Tantalus pillus & Papilio leucothea; P. psittacus \\
\hline Parra chilensis & Phalaena ceraria \\
\hline Otis chilensis & HYMENOPTERA \\
\hline & Cynips rosmarini \\
\hline PASSERES & DIPTERA \\
\hline Columba melanoptera & Tipula moschifera \\
\hline Sturnusloyca & APTERA \\
\hline Turdus thilius; T. thenca; T. curaeus & Aranea scrofa \\
\hline Fringilla barbata; F. diuca & Scorpio chilensis \\
\hline Phitotoma gen.nov. P. rara & $\begin{array}{l}\text { Cancer talicuna; C. xaiva; C. apancora; C. } \\
\text { setosus; C. santilla; C. coronatus; C. } \\
\text { caementarius }\end{array}$ \\
\hline
\end{tabular}


TABLA 2. Clasificaciones del Reino Animal propuestas por Carlos Linneo en su Systema Naturae [ediciones 1 a 13a ]. (Ordenes tratados en el $1^{\text {er }}$ Saggio de Molina subrayados; entre paréntesis se indica el número de géneros en cada Orden).

\begin{tabular}{|c|c|c|c|c|}
\hline \multicolumn{2}{|c|}{ Edición 1 (1735) } & Edic. 10 (1758) & Edic. 12 (1766) & Edic. 13 (Gmelin,1788-) \\
\hline \multirow{2}{*}{\multicolumn{2}{|c|}{$\begin{array}{l}\text { CLASE QUADRIPEDIA } \\
\text { Orden Antropomorpha }\end{array}$}} & MAMMALIA & MAMMALIA & MAMMALIA \\
\hline & & Primates (4) & Primates (4) & Primates (4) \\
\hline \multicolumn{2}{|c|}{$" \quad$ Ferae } & Bruta (5) & Bruta (6) & Bruta (7) \\
\hline \multirow{2}{*}{\multicolumn{2}{|c|}{$\begin{array}{ll}" & \text { Glires } \\
" & \text { Jumenta }\end{array}$}} & Ferae (6) & Ferae (10) & Ferae (10) \\
\hline & & Bestiae (6) & - & - \\
\hline \multicolumn{2}{|c|}{$" \quad$ Pecora } & Glires (6) & Glires (6) & Glires (9) \\
\hline$”$ & - & $\underline{\text { Pecora }}(6)$ & Pecora (6) & Pecora (8) \\
\hline \multirow{2}{*}{$"$} & - & Belluae (2) & Belluae (4) & Belluae (4) \\
\hline & - & Cete (4) & Cete (4) & Cete (4) \\
\hline \multicolumn{2}{|c|}{ CLASE AVES } & AVES & AVES & AVES \\
\hline \multicolumn{2}{|c|}{ Orden Accipitres } & Accipitres (4) & Accipitres (4) & Accipitres (4) \\
\hline \multirow{2}{*}{\multicolumn{2}{|c|}{$\begin{array}{ll} & \text { Picae } \\
" \quad \text { Macrorhyncha }\end{array}$}} & Picae (17) & $\underline{\text { Picae }}$ (22) & Picae (23) \\
\hline & & - & - & - \\
\hline \multicolumn{2}{|c|}{ " Anseres } & $\underline{\text { Anseres }}(11)$ & Anseres (12) & Anseres (13) \\
\hline \multicolumn{2}{|c|}{ " Scolopaces } & Grallae (15) & $\underline{\text { Grallae }}$ (18) & Grallae (19) \\
\hline \multicolumn{2}{|c|}{ " Gallinae } & Gallinae (5) & Gallinae (7) & Gallinae (10) \\
\hline \multicolumn{2}{|c|}{ " Passeres } & $\underline{\text { Passeres }}(11)$ & $\underline{\text { Passeres }}(15)$ & Passeres (17) \\
\hline \multicolumn{2}{|c|}{ CLASE AMPHIBIA } & AMPHIBIA & AMPHIBIA & AMPHIBIA \\
\hline \multicolumn{2}{|c|}{ Orden Serpentia } & Reptiles (4) & Reptiles (4) & Reptilia (4) \\
\hline \multirow{2}{*}{$”$} & - & Serpentes (6) & Serpentes (6) & Serpentes (6) \\
\hline & - & Nantes (6) & Nantes (14) & - \\
\hline \multicolumn{2}{|c|}{ CLASE PISCES } & PISCIUM & PISCES & PISCES \\
\hline \multicolumn{2}{|c|}{ Orden Plagiuri } & Apodes (7) & Apodes (8) & Apodes (12) \\
\hline \multirow{5}{*}{$\begin{array}{l}” \\
” \\
" \\
” \\
”\end{array}$} & Chondropterygii & Jugulares (6) & Jugulares (5) & Jugulares (11) \\
\hline & Branchiostegi & Thoracici (17) & Thoracici (17) & Thoracici (20) \\
\hline & Acanthopterygii & Abdominales (13) & Abdominales (17) & Abdominales (16) \\
\hline & Malacopterygii & Branchiostegi (8) & & Branchiostegi (10) \\
\hline & - & & & Chondropterigii (5) \\
\hline \multicolumn{2}{|c|}{ CLASE INSECTA } & INSECTA & INSECTA & INSECTA \\
\hline \multicolumn{2}{|c|}{ Orden Coleoptera } & Coleoptera (25) & Coleoptera (30) & Coleoptera (55) \\
\hline \multicolumn{2}{|c|}{ " Angioptera } & Hemiptera (8) & Hemiptera (11) & Hemiptera (14) \\
\hline \multicolumn{2}{|c|}{ " Hemiptera } & Lepìdoptera (3) & Lepidoptera (3) & Lepidoptera (3) \\
\hline \multicolumn{2}{|c|}{ " Aptera } & Neuroptera (6) & Neuroptera (7) & Neuroptera (7) \\
\hline$"$ & - & Hymenoptera (10) & Hymenoptera (10) & Hymenoptera (15) \\
\hline$"$ & - & Diptera (10) & $\overline{\text { Diptera (10) }}$ & Diptera (12) \\
\hline$"$ & - & Aptera (14) & Aptera (14) & Aptera (15) \\
\hline CLASE & VERMES & VERMES & VERMES & VERMES \\
\hline Orden & Reptilia & Intestina (8) & Intestina (7) & Intestina (21) \\
\hline & Testacea & Mollusca (14) & Mollusca (18) & Mollusca (31) \\
\hline$”$ & - & Testacea $(16+17)$ & Testacea $(17+19)$ & Testacea $(17+19)$ \\
\hline$”$ & - & $\overline{\text { Lithophita (3) }}$ & Lithophyta (4) & $\overline{-}$ \\
\hline$"$ & Zoophita & Zoophita (11) & Zoophyta (15) & Zoophyta (15) \\
\hline & & & & Infusoria (15) \\
\hline
\end{tabular}


TABLA. 3. Citas de autores en Linneo, ed. 12, 1766. (Visite www.animal.base.de por "Abreviaciones" y referencias completas).

MAMMALIA

Precedentes: Gesnerus, Aldrovandus, Jonstonus

Praesentis: Rajus, Brisson, Houttinus

AVES

Veterum: Bellonius, 1557, Gesnerus 1555, Aldrovandus 1599,

Novas M arcgravio, 1648, Willugby 1676, Rajo 1713, M arsiglius 1726

Recentiores ilustradores: Frischius 1734, Albinus 1731, Catesby, 1731, Edwards 1745

\section{PISCES}

Practici: Bellonius 1552, Rondeletius 1554, Salvianus 1554, Gesnerus 1558, Aldrovandus 1605, Willugbaeus 1685, Rajus 1710, Seba, 1760

Teoretici: Artedi!, Nos, Gronovii, Hasselquist (observan radios pinnae) Valentynus, Catesbaeus, Garden,

AMPHIBIA

Amphibiologi: Seba, Catesbaeus, Gronovius \& Garden

INSECTA (larga lista)

Veteres: M onffetus, Aldrovandus, Jonstonus

Philosophi: Swammerdam, Reaumur!, De Geer, Bonnet

Descriptores: Rajus, Fauna Svecica, M useum Reginae (ambas de Linneo)

Monographi: Lister, Schaffer, Clerck

Systematici; Sistema naturae, Poda, Sultze, Geoffroa, Scopoli, Gronovius

\section{VERMES}

Molluscorum: praetere Bobadschium pauci

Testaceorum primarii: Bonannus, 1681, Listerus 1685, Rumphius 1705, Petiverius 1702, Gualterius 1742, Argenville 1742, Regenfusus 1758 ( $h$ )

Nec non Columna, Barrelierus, Plancus, Kleinius, Ginannus, Adansonus, Seba

Lithophytorum: Marsiglius, Donatus

Zoophytorum: Ellisius, Basterus 


\section{LAS OBRAS SOBRE CIENCIAS NATURALES Y EL DESARROLLO DE LA ZOOLOGIA}

Hay varias razones importantes que permitieron que un clérigo que llegó a tomar lecciones de historia natural a Bolonia, sin mayor formación naturalista y sin colecciones o ejemplares a la vista, llegara a describir especies chilenas para permitir incorporarlas al conocimiento europeo de la historia natural. Una es su conocimiento del latín y el griego, resultado de su formación religiosa. Con ese bagaje, J.I. Molina y sus cofrades de la Compañía de Jesús, al llegar a Italia, luego de la expulsión de los jesuitas de América, debieron aprender rápidamente italiano como lo hace ver el llamado "autor anónimo" (en nuestra opinión Felipe Gómez de Vidaurre) en la introducción al Compendio sulla storia geographica..., publicada en esa lengua en 1776. Indudablemente, lo mismo debe haber ocurrido con el abate. Molina, aunque Hanisch (1985:3) en uno de sus últimos artículos sobre este abate afirma que en Chile había aprendido la lengua italiana “... como todas las demás que supo: latín, griego y francés”. ¡Extraordinaria formación general, para un hombre joven que a sus treinta y tres años llega a Bolonia, si eso fuera efectivo! Al respecto, llama la atención que Molina, a diferencia de Ovalle, o Gómez de Vidaurre, nunca escribiera sus obras también en español, o en latín como Linneo y otros verdaderos naturalistas lo hacían en esa época, sino sólo en italiano.

Otra razón favorable es que se enfrentó a una taxonomía para los animales conocidos (y las plantas), que era aún relativamente simple en su época, basada en unos pocos caracteres diagnósticos descritos en el sistema de Linneo y como tales cambiantes. Sin embargo, la razón más importante, es que el abate tuvo a su disposición descripciones generales de todas las especies que describió, las que se encontraban en el Compendio anónimo, algunas en las ediciones de la obra de Linneo, o en los manuscritos inéditos que el conoció, escritos por otros clérigos naturalistas anteriores a /o contemporáneos de él, y los informes publicados por los primeros viajeros europeos que llegaron a nuestras costas.

Por otra parte, el conocimiento general de los grandes grupos animales y las especies más comunes, ya había sido avanzado en las primeras grandes publicaciones sobre los grupos más importantes aparecidas durante los siglos XVI, XVII y comienzos del XVIII. Todas, por autores mencionados de manera especial por Linneo en la edición 12 de su Systema Naturae (1776), como complemento a las importantes características morfológicas generales ya conocidas para cada una de las distintas clases animales: Mammalia (vol. 1, p. 21), Aves (vol. 1, p. 114), Amphibia (vol. 1, p. 348), Pisces (vol.1, p. 421), Insecta (vol. 2., p. 535) y Vermes (vol. 2, p. 1071). La lista de los autores indicados por Linneo en latín se presenta en la Tabla 3, pero los nombres completos de los autores, título, año y contenidos de cada obra se puede 


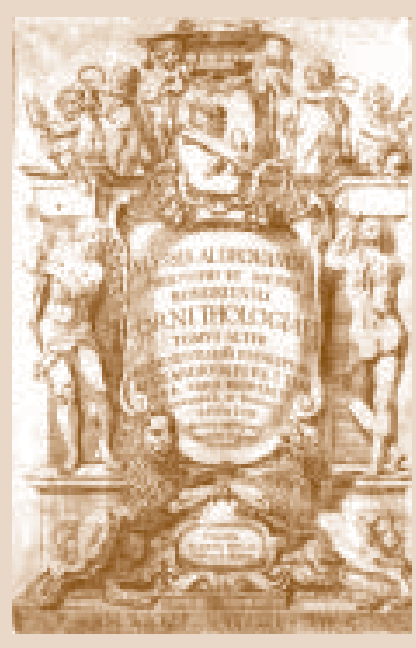

${ }^{[10]}$ Obra de Aldrovandi.

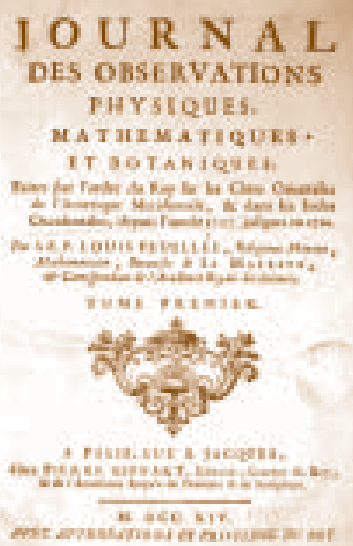

${ }^{[11]}$ Obra de Feuillée. consultar hoy en día online, en el importante programa "Animal Base. Early Zoological Works", desarrollado por la Universidad de Gotinga (Goettingen), evitando el repetirlos aquí: http://www.animalbase.uni-goettingen.de/ zooweb/servlet/AnimalBase/search

¿Cuáles de estas obras conoció y consultó Molina para escribir su primer Saggio? En el Anexo 1 se presenta, a su vez, una lista de los autores y referencias citadas en su texto. Se constata que las publicaciones consultadas son escasas y que la mayoría son referencias a observaciones hechas por viajeros naturalistas que acompañaron a las primeras exploraciones a las regiones australes del hemisferio sur, principalmente aledañas al Estrecho de Magallanes. Es extraño, por ejemplo, que no haya citado a los especialistas que Linneo identifica con los distintos grupos animales mejor estudiados en su época, sobre todo porque tiene que haber conocido algunas de las obras existentes en Bolonia, cuna de Ulisse Aldrovandi, autor prolífico, y creador del Museo y de la Biblioteca que formó en la Universidad de Bolonia, que años más tarde lo acogió a él ${ }^{[10]}$. Pero Molina tampoco hace referencia a las obras de éste, ni a las de los otros naturalistas renombrados citados por Linneo, y que en 1782 eran ya de consulta obligada y análisis en los estudios de nuevas faunas.

\section{EL CONOCIMIENTO DE LAS ESPECIES CHILENAS POR AUTORES ANTERIORES}

Con anterioridad a Molina, no hay trabajos taxonómicos de autores extranjeros sobre animales chilenos, salvo por unas pocas especies cosmopolitas descritas por Linneo. Sin embargo, hubieron dibujos y descripciones generales de animales (y plantas) hechas por los primeros viajeros que se aventuraron por el llamado Mar del Sur.

En el Prefacio de su Ensayo... de 1782 (impropiamente denominado Compendio en su traducción ) cita entre sus fuentes informativas generales a "los viajeros instruidos que llegaron a Chile", pero agrega que "sus noticias son demasiado sucintas...”. Nombra en primer lugar al religioso Louis Feuillée (1660-1732), quien en 1724 publicó en 3 volúmenes su conocida obra Journal des observations physiques, mathématiques et botaniques... [11] en la que describe algunas plantas y animales (Anexo 1). Luego, menciona a "los nacionales" ("naturales" (!), en la traducción de Arquellada Mendoza) que habían publicado historias que circulaban como manuscritos que Molina consultó, pero que no habían sido aún publicadas cuando escribía su libro. Nombra a tres autores: Pedro de Figueroa y los abates Miguel de Olivares y Felipe Vidaurre (Felipe Gómez de Vidaurre). De Vidaurre, escribe con encomio que se "dedicó" principalmente "a manifestar las producciones chilenas y sus usos, lo que ha hecho con gran inteligencia de la materia que trata”. 
En el mismo Prefacio, después de la obra del jesuita Alonso de Ovalle, publicada en italiano y español en 1646 , cita un Compendio anónimo publicado en lengua italiana en 1776, de cuyo autor, entonces desconocido, dice que "da una noticia más completa de Chile que aquellas otras impresas, particularmente respecto de la Geografía y la Historia Natural". Esta obra, como discutimos más adelante, fue fundamental en la redacción de su propio primer Saggio.

En el texto propiamente tal, y en relación sólo a unos pocos grupos animales, menciona, principalmente como notas a pie de página, observaciones o comentarios que aparecen en las obras o relatos en francés o traducidos al francés de: Amedée F. Frezier (1716) ${ }^{[12]}$; Peter Kolbe (1743); Jorge Juan y Antonio de Ulloa (1748) ${ }^{[13]}$ en español y francés; Lord (George) Anson (1750) en inglés y francés; George W. Steller (1751), en latín; John Hawkesworth (1774, en la traducción francesa que incluye los relatos de los viajes de James Cook, Samuel Wallis, John Byron y Philip Carteret también citados); Dom Pernetty (1770-74), en francés, y Louis Antoine de Bougainville (1771), que incluye los relatos de Nicolas-Pierre Duclos-Guyot y Francois Ch. de la Giraudais, sus capitanes (Anexo 1). Respecto a interpretaciones antropológicas cita también a Cornelio Pauw (1769). Ninguno de ellos es mencionado en su "Catálogo de los Escritores de las cosas de Chile", aparecido como parte de su Saggio sulla storia civile... (Ensayo sobrehistoria civil), publicado en 1787.

Hay otro trabajo no publicado en la época que aquí tratamos que Molina reconoce que conoció como manuscrito: el del también jesuita Diego de Rosales. Pero en el hecho, las obras que le sirvieron de base son principalmente las de Ovalle ${ }^{[14]}$, Olivares y de Gómez de Vidaurre. Aunque las dos últimas fueron publicadas tardíamente por el ilustre bibliógrafo don José Toribio Medina, permiten conocer cuánto de esos textos usó Molina en la descripción de sus nuevas especies animales, de lo que él mismo, urgido por sus críticos, deja constancia posteriormente, al menos para algunas especies, en el texto de la segunda edición "revisada" de su Saggio sulla storia naturale del Chili, escrita en italiano y aparecida también en Bologna en el año $1810^{[15]}$. Esta obra fue sólo hace un par de décadas traducida al español por R. Jaramillo (1985).

Paradójicamente, en esta "revisión" de su primer Saggio... de 1782, no describe ninguna especie nueva. Sólo repite sus primeras descripciones y copia a destajo nombres de especies descritas de América y el océano austral (pero no de Chile), registradas por Gmelin en la edición 13 del Systema Naturae ya citada, en la quetambién se compila por primera vez a la mayoría de las especies chilenas descritas por M olina, en 1782. Dejándose llevar por este importante reconocimiento, introduce desgraciadamente también numerosos nombres de especies desconocidas, principalmente invertebrados, sin descripciones, que sugiere ser "afines" a otras ya conocidas (e.g. Halyotis

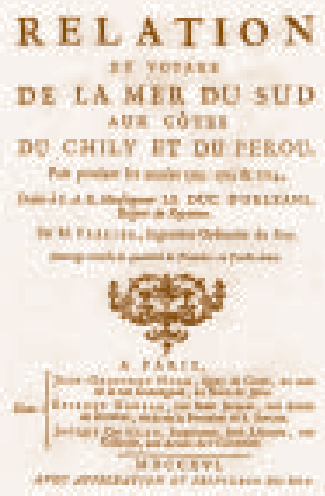

${ }^{[12]}$ Obra de Frezier.

REL_ACION HHSTORICA pax riasa

A LA AMIULCA MERIDOCONAI

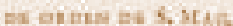

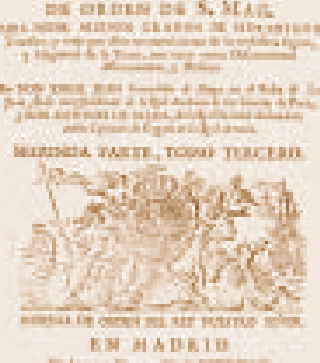

EMA A D D IO

${ }^{[13]}$ Obra de Ulloa.

H I S T O R I C A

R. I. L \& C I O N

an Bopto chrs.

"-

4 visure crios

IFS C HRISTO

Dios HONBNE

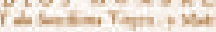

aA in is

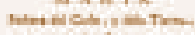

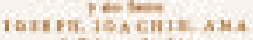

inllow in ap

ot onso n oraLLE

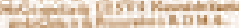

genes

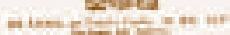

${ }^{[4]}$ Obra de Ovalle. 
SACEIO

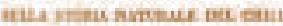

G10 IGNazio MoLiNa

srovar rerkese

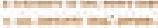

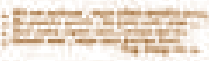

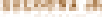

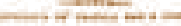

${ }^{15}$ Saggio de 1810.

dolkiscion

IIISIURIIIIIIIS II, IIIIII

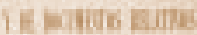
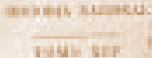

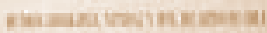
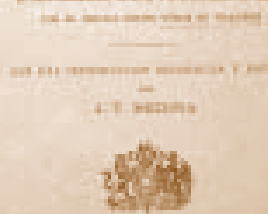

$\sin =0$

tins

6] Historia... de Gómez de Vidaurre. tesselata al. australis; Conus punctatus al. M agellanicus, etc.), aparentando un mayor conocimiento de la fauna chilena terrestre y marina que indudablemente no podía tener, y de especies que nunca había visto, nombres que obviamente nadie consideró, y que por no tener descripción o figura alguna, pasaron al olvido. Las dudas válidas sobre esta obra fue indudablemente la causa de que no se tradujera en su tiempo a otras lenguas ${ }^{[15]}$.

En su primer Saggi o... de 1782, como ya hemos mencionado, copió también los nombres autóctonos y las descripciones generales de todas las especies mencionadas por los primeros autores que escribieron sobre especies chilenas como son (en orden de fechas): Ulloa, Frezier, Feuillée, Ovalle, Rosales, Olivares y especialmente los llamados Compendio anónimo de 1776, y la Historia geográfica, natural y civil del Reino de Chile, escritas por Felipe Gómez de Vidaurre. Esta última fue consultada por Molina como un manuscrito (MS) no publicado, como el mismo menciona en su introducción al primer Saggio... , pero usado ya entonces como un valioso documento de su tiempo, el que, afortunadamente para la historia de la ciencia, fue rescatado y publicado en Chile por J.T. Medina en el año $1889^{[16]}$.

Que Felipe Gómez de Vidaurre fue también el autor del Compendio anónimo, se concluye no sólo de la corroboración dada por su primer traductor al alemán (injustamente vilipendiado como ignorante por algunos biógrafos de Molina), sino también por la importante opinión apoyando esta conclusión adelantada por José Toribio Medina al publicar en 1889 su manuscrito de la H istoria geográfica natural y civil... escrita por Gómez de Vidaurre en español y, especialmente, por su propio testimonio reclamando su autoría en dos cartas que transcribe Hanisch (1976: 176). Pero, incomprensiblemente este conocido autor mantiene la duda sobre el anónimo, sólo sugiriendo que tales cartas abren un capítulo de encuentro personal, y no aclarando que M olina nunca se atrevió a reconocer en sus escritos que él fuera el autor del Compendio. La observación de que el mismo Molina habría reconocido esto en la traducción al francés de su Saggio... por M. Gruvel (1789: ix) es totalmente infundada. Este sólo repite que "el traductor alemán de este compendio lo ha atribuido sin fundamento al Abate Vidaure (sic)" (Traducción).

Por otra parte, una comparación de los nombres de las especies y sus descripciones generales publicadas por Gómez de Vidaurre en el Compendio... de 1776, escrito originalmente en italiano, y en la Historia... mencionada, publicada en 1889, como hemos hecho para esta publicación, lleva a la misma conclusión, corroborando de paso, que la mayoría de las descripciones de Molina son, con muy pocas excepciones, copias a menudo textuales o sólo arregladas en su redacción, de las descripciones de las especies que aparecen principalmente en estas dos obras de Gómez de Vidaurre.

Sin embargo, es también justo reconocer, que hay descripciones en obras anteriores de otros autores que a su vez han sido copiadas en las tres obras 
que analizamos, demostrando que ya a fines del siglo XVIII, el conocimiento aportado por las primeras expediciones europeas al Mar del Sur y a las tierras australes ya había comenzado. Además, los primeros naturalistas que adoptaron el sistema binomial de Linneo para describir a las especies, seguían la práctica de publicar una lista de nombres y referencias de obras consultadas con descripciones y figuras (la "sinonimia") como era ya práctica en las monografías publicadas antes de Linneo, lo que fue sólo tardía y vagamente reconocido por nuestro compatriota en su Saggio... de 1810. El honor de proponer un nombre científico a perpetuidad, reconociendo que ya había sido descrito de manera general por otro autor, no fue minimizado entonces, ni lo es en la actualidad. Es más, la publicación de Linneo desató en la historia de la zoología una verdadera carrera por describir nombres de especies nuevas, muchas de ellas dibujadas exquisitamente en obras que daban a conocer en descripciones generales la fauna y flora de lugares ignotos. Un magnífico ejemplo de ello es la famosa obra del acaudalado boticario de Amsterdam Albertus Seba (1734-1765), Locupletissimi Rerum Naturalium Thesauri... en 4 volúmenes, conteniendo 446 láminas en color de animales y plantas de las Indias Orientales, Europa y otras regiones, que Seba compraba y coleccionaba, y que dio origen a muchos tipos de nuevos animales y plantas descritas por Linneo y autores posteriores ${ }^{[17,18]}$.

Los problemas creados por la incomprensible parquedad o malicia de Molina para ocultar sus fuentes de consulta ocurrieron en todos los grupos animales descritos por él. Y hubiera permanecido así si no fuera por la crítica de Brogniart (véase a Hanisch, 1976:129-130), a la que respondió sólo en parte dando algunas referencias en la segunda edición "revisada" de su Ensayo de 1810, pero ocultando otra información que motivó otras críticas posteriores muy fuertes como las de Alcide d'Orbigny (1835-1843:51 y siguientes $)^{[19]}$ y aun de Claudio Gay (1854, vol. 8, Zoología: 472). A la luz de estos antecedentes se justifica una discusión extensa de las especies descritas en el Saggio... de 1782, lo que será objeto de otra publicación, sin embargo, consideramos procedente comentarlas brevemente a continuación.

\section{LA COMPLEJA CLASE DE LOS VERMES EN MOLINA, 1782 (MOLLUSCA Y TESTACEA)}

En la época de Molina, éste era el grupo más diverso y mal conocido por los naturalistas. Animales tan conspicuos como el piure, los erizos, el picoroco, observables en las rocas de cualquier playa junto a caracoles y bivalvos, ya individualizados y descritos en algún detalle por los primeros clérigos-naturalistas nacionales (Ovalle, Rosales, Olivares, y en particular Gómez de Vidaurre en el Compendio de 1776), representaron uno de los aportes más novedosos al conocimiento internacional de los invertebrados marinos chi-
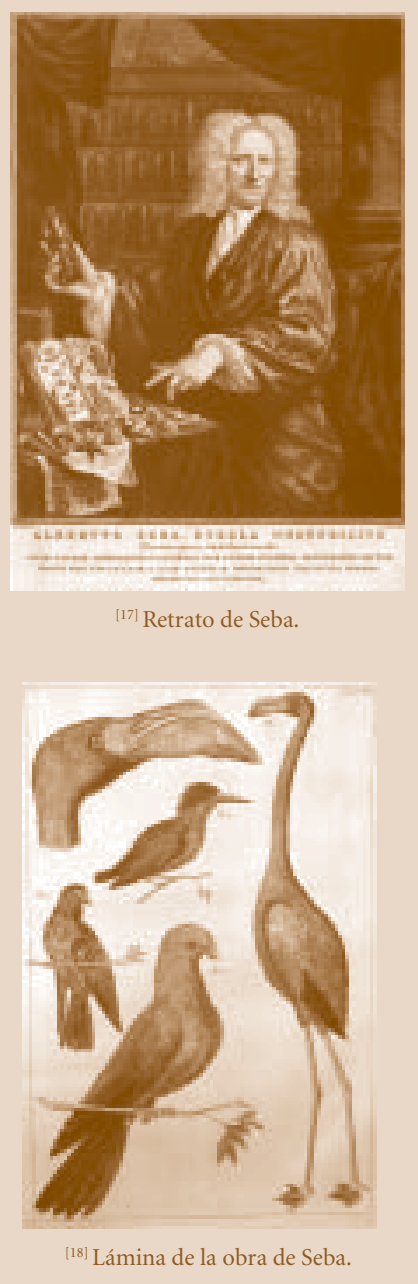

TOYIGE

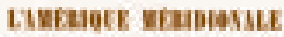
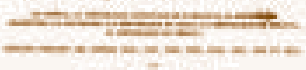

chenservakatez

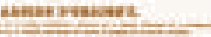

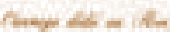
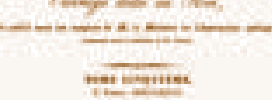

thencinas

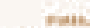

$\min$

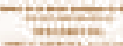

riment

${ }^{19]}$ Obra de d' Orbigny. 
RELATION

DES VOYAGES rxespon ras giass

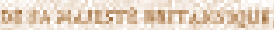

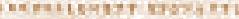

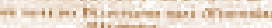

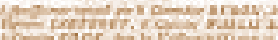

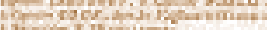

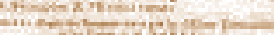

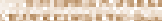

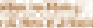
inters

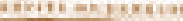

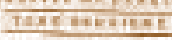
-

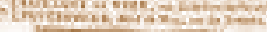

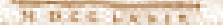

${ }^{[20]}$ Obra de Hawkesworth.

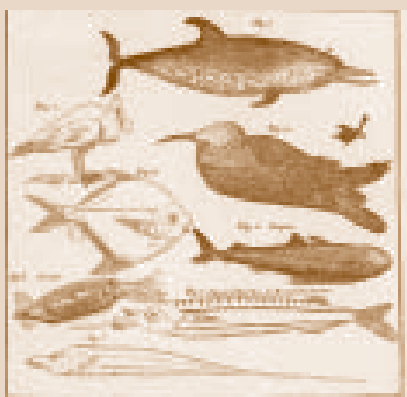

[21] Lámina de Pernetty.

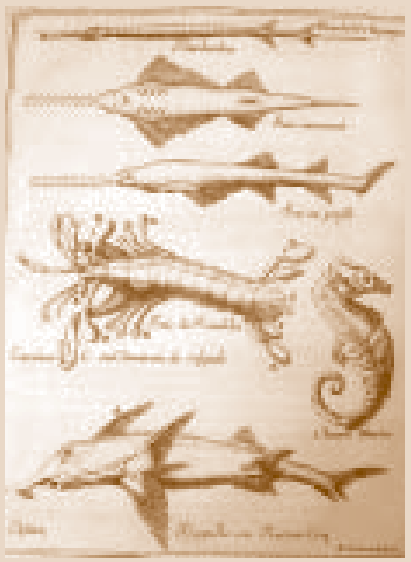

${ }^{[22]}$ Lámina de Ulloa (pejegallo). lenos, debido a sus formas bizarras, gran tamaño, abundancia e importancia para el consumo popular. Molina tomó de ellos sus descripciones, incluyendo la del "pulpo", una especie irreal de seis patas mezcla de pulpo y de insecto (el "palote") que obviamente era un engendro inexistente que denominó "Sepia exapodia", originalmente mal descrito por Frezier (1716). De igual manera describió otras dos especies irreconocibles (S.unguiculata, S.tunicata) basadas una en un relato de Banks $(1777)^{[20]}$ y la otra, en un pequeño ejemplar parcialmente digerido, que Pernetty (1770) encontró en el Atlántico en el estómago (!) de un "bonito" (Anexo 1$)^{[21]}$; pero Molina no informa sobre esto.

Con todo, no deja de ser significativo que de un total de 15 especies descritas por Molina 10 son en la actualidad especies válidas (Tabla 1).

\section{LA CLASE PISCES EN MOLINA, 1782}

A fines del siglo XVIII éste era uno de los grupos menos conocidos para la ciencia en el Mar del Sur. En el Compendio de 1776, Gómez de Vidaurre afirmaba que en Chile "se encuentran más de 60 especies en su mayor parte diferentes de las de Europa", y en su MS, publicada posteriormente en 1889, concluía que "... los pescadores reconocen 76 especies". Molina en su Saggio... de 1782 reconoce el mismo número y describió 11 especies, de las cuales cuatro son válidas pero sólo tres llevan su nombre (Tabla 1). Ya en esta época, las características para diferenciar las especies introducidas por Linneo en el Sistema Naturaey, en el hecho, por autores anteriores, requería de dibujos precisos y observaciones complejas; por ejemplo, la necesidad de contar el número de radios y espinas de las aletas y de los arcos branquiales como parte importante de cada descripción.

Pero, ¿cómo pudo Molina determinar el número de branquispinas, y radios de las aletas de las especies que describió con nombre científico, sin dibujos o ejemplares para examinar, si no fuera inventando un número afín a las especies descritas en los géneros de Linneo en que incluyó a las especies chilenas? Los otros caracteres generales de sus diagnosis los copió de las descripciones generales dadas para las mismas especies por Gómez de Vidaurre en sus dos obras, y en el caso del tollo de Juan Fernández por Ulloa (1748).

No habían figuras de peces chilenos publicadas por autores nacionales y en la literatura extranjera sólo se conocía la exacta figura del "pejegallo", la Chimaera callorhynchus Linné, de su Orden Nantes (Amphibia), especie cosmopolita dibujada en una de sus láminas por Frezier (1776, lám. XVII) ${ }^{[22]}$, naturalmente sin nombre científico. Molina (1782) no menciona ni copia la figura de esta especie, $u$ otras (Anexo 1). 
Este es un grupo que en los tiempos de Molina era mucho mejor conocido por los naturalistas y viajeros que los Vermes o los Peces. Respecto al número de especies, Gómez de Vidaurre dice en el Compendio... de 1776 que las aves que pudo observar "tanto terrestres como acuáticas" son 72 "con un número sorprendente de individuos"; en su Historia... de 1889 repite el mismo número. Molina en su Saggio... de 1782 eleva el número de especies de aves terrestres y acuáticas conocidas a 135, agregando que sólo el género de las gaviotas (¡usa el nombre técnico Larii en el original italiano que Arquellada Mendoza traduce como "golondrinas de mar"!) "contiene 26 o 27 especies distintas"; obviamente una exageración.

De las 34 especies que describió, 20 son buenas en la actualidad (Tabla 1), incluyendo un género nuevo Phytotoma para la "rara" (Phytotoma rara), cuya descripción atribuye Molina en el Saggio de 1810 a Gómez de Vidaurre. Sin embargo, ocurre aquí la misma copia de las descripciones de los autores antes mencionados, en particular de Gómez de Vidaurre (1889) que Molina conoció como MS antes de su publicación y menciona en la segunda edición de su Saggio... de 1810.

Este es el único grupo para el que se habían publicado dibujos atribuidos a especies chilenas; por ejemplo, los de la lámina del Compendio anónimo ilustrando a figuras de un pingüino, un picaflor, el queltehue, un flamenco y el cóndor ${ }^{[23]}$, todas consideradas hasta ahora dibujadas por su autor (Gómez de Vidaurre). Desgraciadamente, ninguna de ellas representa a especies chilenas sino que fueron copiadas del Gazzettiere Americano (1763) en donde se publicaron para ilustrar otras especies americanas, pero no chilenas y cuyo autor anónimo las copió a su vez de G. Edwards $(1743,1750)^{[24,25]}$. ¡Molina no comenta estos errores!

Copiar descripciones y figuras de otros autores aparecía ya como una práctica de la que Gómez de Vidaurre tuvo que echar mano para justificar las especies que describió en el Compendio..., pensando que eran las mismas para otros países americanos, pero que desgraciadamente no eran las especies chilenas ${ }^{[26]}$. ¿Por qué Molina no criticó las figuras del Compendio... y no dibujó sus especies si las conocía tan bien?

\section{LA CLASE MAMMALIA EN MOLINA, 1782}

Los mamíferos es el otro grupo mejor conocido por lo clérigos-naturalistas mencionados. Gómez de Vidaurre dice en el Compendio... de 1776, que exceptuando a las especies europeas, en Chile hay 36 "especies discernibles". Molina (1782) repite el mismo número, e indudablemente, este conocimiento

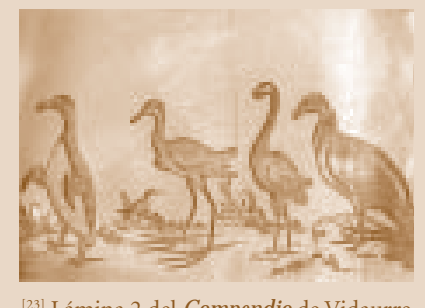

[23] Lámina 2 del Compendio de Vidaurre (Aves).
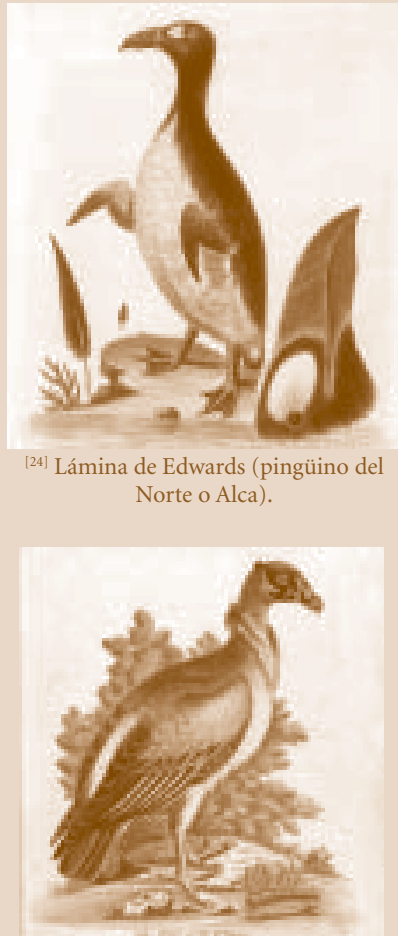

${ }^{[25]}$ Lámina de Edwards (zopilote).

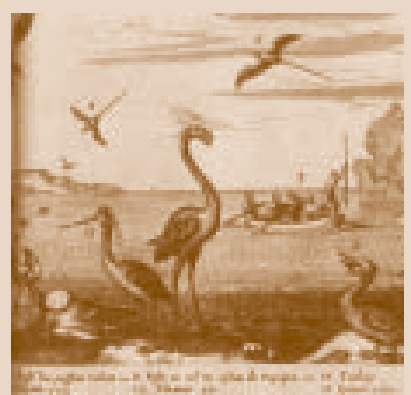

[26] Lámina de Du Tertre (flamenco).

$101 \mid \begin{aligned} & \text { Atenea } 495 \\ & \mid \text { I Sem. } 2007\end{aligned}$ 


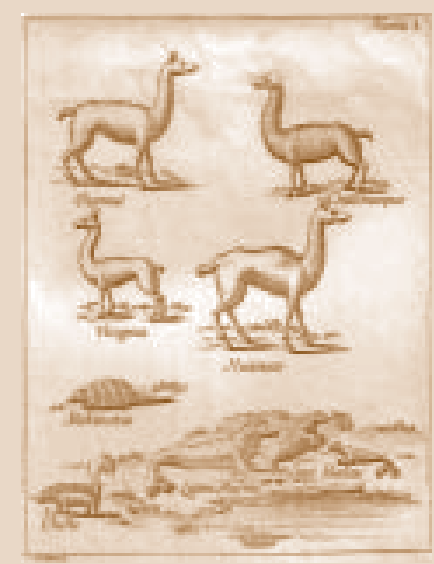

[27] Lámina 3 del Compendio de Vidaurre (Mamíferos).

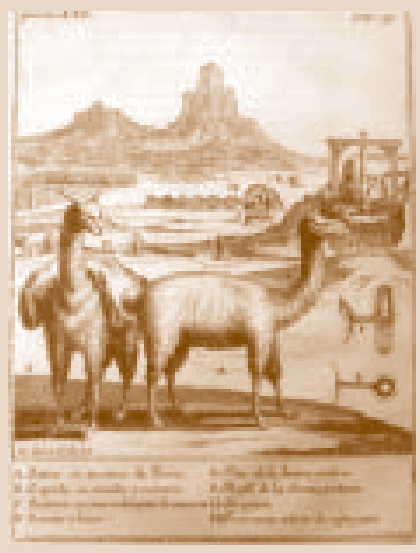

${ }^{[28]}$ Lámina del Chilihueque de Frezier. general le permitió describir 29 especies, de las cuales hay 16 consideradas buenas en la actualidad que llevan su nombre (Tabla 1).

Pero Molina tampoco ilustra al menos a algunas de estas especies, ni hace referencia en el texto a las que habían sido publicadas en la lámina 3 del Compendio ${ }^{[27]}$. Tampoco menciona las dos láminas de Ulloa (1748), en que éste presenta a tres tipos ("especies") de lobos, a un pájaro niño y a las conchas de "loco" y los conchales; ni a la lámina 22 de Frezier (1716) en que muestra un excelente dibujo de la llama o Chilihueque ${ }^{[28]}$; o a la lámina 3 del Compendi ${ }^{[27]}$ en que se ilustran una figura equivocada del huemul, junto a otras del huanaco, la llama, la vicuña, un quirquincho, el chingue, y las mismas tres especies de lobo marino ilustradas por Frezier (1716). Ni menos las figuras de Pernetty (1770) del elefante marino, del león marino y de otras especies, todas figuradas en sus distintas láminas. Incomprensible razón la de Molina para ignorarlas, y no incorporarlas a la lista de referencias (sinonimia) siguiendo el ejemplo de las especies de Linneo y otros autores, que siempre las incluían al describir nuevas especies basadas en referencias nobinomiales.

\section{EPILOGO}

Juan Ignacio Molina, el abate, pasó ya a la posteridad al describir con género y especie a animales característicos de la costa chilena y el Mar del Sur, y se justifica que la Biblioteca del Bicentenario haya publicado un facsímil en español de la obra que permitió tal reconocimiento a nivel mundial, pero se debió haber cautelado sobre las diferencias existentes con el original italiano.

Por otra parte, como se ha demostrado en este resumido análisis de la proyección zoológica de su obra, el denominado Compendio anónimo de 1776, injustificadamente atribuido a Molina y cuyo autor a la luz de un análisis ecuánime resulta ser su contemporáneo, el jesuita Felipe Gómez de Vidaurre, reclama el mismo honor. Así lo reconoció ya el renombrado historiador y editor José Toribio Medina, al publicar en 1889 el manuscrito de la H istoria geográfica, natural y civil del Reino deChile, escrita por Gómez de Vidaurre. Haciendo ver la similitud de los dos textos (como aquí hemos hecho), y reconociendo que esta Historia... presenta, además, el mismo título y las mismas láminas que el Compendio dela historia geográfica, natural y civil del Reyno de Chile, de 1776, concluye estar convencido ("lo creemos ahora", dice) que ...

á él le cabria el envidiable honor de haber dado a conocer en Europa á una nación que era al fin su patria y que tan desfigurada, como lo ha 
dicho, se notaba allí, cuando su duro destino lo llevara á aquellas remotas tierras (1889, Tomo 1, Introducción: xviii).

\section{LITERATURA CITADA}

${ }^{[*}$ Indice de las reproducciones de obras que aparecen en el margen de las páginas de esta contribución.

Anónimo. 1878. Compendio dela historia geografica, natural i civil deChile. (Publicado Anónimo en Bolonia en 1776 i traducido por Narciso Cueto) In: Luis Montt. Colección de Historiadores de Chile y documentos relativos a la Historia Nacional. Tomo XI: 185-304. (sin láminas del original), Santiago de Chile (véase Vidaurre).

Anónimo. 1762-63. The American Gazetteer, containing a distinct account of all the parts of the New World. London, printed for A. Miller.

.1763. II Gazzettiere Americano. Contenente un distinto ragguaglio di tuttele parti del nuovo mondo della loro situazione, clima, terreno, prodotti, stato antico e moderno, merci, manifatture, e commercio con una esatta descrizione delle città, piazze, porti, baje, fiumi, laghi, montagne, passi, e fortificazioni ... Tradotto dall'Inglese e arricchito di Aggiunte, Note, Carte e Rami. Vol.1, 216 pp.; vol. 2, 256 pp.; vol. 3, 253 pp. Livorno, Marco Coltellini.

Brisson, Mathurin-Jacques. 1756. Regnum animalein classes IX distributum. Sive Sinopsis M ethodica... cum brevi cujusque speciei descriptione, citationibus auctorum, etc. Le regne animale divisé en IX. Classes. Paris, 382 pp.

D’Orbigny, Alcide. 1835-1843. Voyage dans I'Amérique M éridionale... Exécuté pendant les années 1826,1827,1828,1829,1830,1831,1832 2t 1833. Tome cinquième, 3.e Partie: Mollusques. Paris. Chez P.Bertrand, éditeur.

Edwards, George. 1743. A natural history of birds. M ost of which have not been figur'd or describ'd, and others very little known from obscure or too brief descriptions without figures, or from figures very ill design'd. Containing the figures of sixty birds and two quadrupedes... Vol. 1, i-xxiv, 1-53. London, College of Physicians in Warwick-Lane.

1750. A natural history of uncommon birds, and of some other rare and undescribed animals, quadrupedes, reptiles, fishes, insects, \& c. Exhibited in two hundred and ten copper-plates, from designs copied immediately from nature, and curiously coloured after life. With a full and accuratedescription of each figure. Part III: 106-157. London, ibid.

Feliú Cruz, Guillermo. 1966. H istoria de las Fuentes de la bibliografía chilena. Ensayo crítico. Tomo 1, 384 pp. Santiago de Chile, Biblioteca Nacional.

Gay, Claudio. 1854. H istoria física y política de Chile. Zoología, Tomo 8, 499 pp.

Gmelin, Johann Friedrich. 1788-1792. Caroli A. LinnéSystema Naturaeper Regna Tria Naturae... Editio Decima Tertia, Aucta, Reformata. Vol. 1, tomos 1-6, 4120 pp. Lipsiae, Impensis Georg. Emanuel. Beer.

Gómez de Vidaurre, Felipe (ver también Vidaurre). 1889. Historia geográfica, natural y civil del reino deChile. Publicada con una introducción y notas por 
J.T. Medina. Tomo I. In: Colección de Historiadores de Chile y de documentos relativos á la historia nacional. Tomo XIV: 1-357, Santiago de Chile: Imprenta Ercilla.

Hanisch, Walter. 1976. Juan Ignacio M olina, sabio de su tiempo. 178 pp. Ediciones "Nihil Mihi", Santiago de Chile.

. 1986. "El ambiente chileno y europeo en la formación de Juan Ignacio Molina". Universum, $\mathrm{N}^{\circ} 1$ (Talca): 5-16.

Jaramillo, Rodolfo (véase J.I. Molina, 1985).

Molina, Juan Ignacio. 1782. Saggio sulla storia naturaledel Chili. 349 pp. Bologna, Stamperia di S. Tommaso d'Aquino.

. 1786. Versuch einer Naturgeschichte von Chili. Von Abbé J.Ignaz Molina.Aus dem I talianischen übersetz von J.D. Brandis. M it einer Landkarte. 328 pp. Leipzig, bey Friedrich Gotthold Jacobaer.

. 1789. Essai sur I'H istoire N aturelle du Chili par M. l'abbé Molina. Traduit de l'Italien, \& Enrichi de notes. Par M. Gruvel, D.M. 351 pp. Paris, Chez Née de la Rochelle, Libraire.

. 1808. The geographical, natural, and civil history of Chili... With notes from the Spanish and French versions, and Appendix... Translated from the original Italian by an American gentleman. 2 vols. Middletown, Connecticut, for I. Riley.

. 1809. Ibid. With two Appendixes by the English editor. 2 vols. London. Printed for Longman, Hurst, Reese and Orme. . 1878. Compendio de la historia geográfica, natural i civil del reino de Chile. Escrito en italiano por el abateD. Juan I gnacio M olina, Primera Parte, que abraza la historia geográfica y natural. Traducida en español por don Domingo José de Arquellada Mendoza. Individuo de la Real Academia de Buenas Letras de Sevilla, i Maestrante de Ronda. In: Luis Montt. Colección de Historiadores de Chile y de documentos relativos a la Historia Nacional. Tomo XI: 305-522 (con notas). Santiago de Chile.

. 1985. Ensayo sobrela historia natural deChile. Traducción del Prof. D. Rodolfo Jaramillo, de la Academia de las Ciencias del Instituto de Bolonia. Ediciones Maule, 383 pp., 1 mapa. Santiago de Chile.

Seba, Albertus. 1734-1765. Locupletissimi Rerum Naturalium Thesauri Accurata Descriptio, et Iconibus Artificiossimis Expressio, per Universam Physices Historiam... 4 vols. (3 y 4 póstumos). Amstelaedami, apud JanssonioWaesbergios, \& J. Wetstenium, \& Gul. Smith.

Vidaurre, o Gómez de Vidaurre, F. 1776. Compendio della storia geografica, naturalee civile del regno del Chile. 245 pp., 1 mapa, 10 láms. Bologna, Nella Stamperia di S. Tommaso d'Aquino.

natürliche und bürgeliche Geschichte des Königreichs Chile, aus dem Italianischen ins Deutsch übersetzt von E.J. [agemann]., 208 pp. Mit einer charte. Hamburg, bey Carl Ernst Bohm. 


\author{
ANEXO I \\ Referencias del primer Saggio de Molina de 1782
}

Acosta, José de 1590. Historia natural y moral delas Indias. Sevilla.

Anson, Lord George. 1750. Voyage à la M er du Sud et voyage autour du monde faits dans les années 1740 à 1744. Paris. (U otras ediciones posteriores en francés).

Buffon, George Louis Leclerc, Comte de 1749-1789-1825. Histoire naturelle général et particulière avela description du cabinet du roi. Paris.

Carteret, Philip. An account of a voyage round the world, in the years 1766,1767, 1768, and 1769. (Véase Hawckesworth, 1774).

Clavijero, Francisco Javier. 1780-1781. H istoria de M éxico. Bolonia.

Byron, John 1767. Voyage autour du mondefair en 1764 \& 1765 sur levaissau de guerre anglais le Dauphin... dans lequel on trouve une description exacte du D etroit de Magellan, etc... Paris.

. 1769. Viajedel comandanteByron alrededor del mundo... Traducción del Dr. Casimiro Ortega. Madrid.

Cook, James 1777. A voyagetowards the South Poleand round theworld. Performed in his M ajesty's ships the Resolution and the Adventure. In the years 17721775. London, 2 vols.

. 1777. Journal du second voyage du CapitaineCook, sur les vaisseaux

la Résolution et l'Aventure, entrepris par ordre de sa majesté britannique dans les années 1774 \& 1775. Amsterdam y Paris, Pissot \& Nyon, en -8 de XIX (1)546 pp. et une planche dépliante de l'hémisphère sud.

_. Voyage dans I'hémisphère austral et autour du monde fait sur les vaisseaux deroi l'Aventure \& la Résolution, en 1772, 1773, 1774 \& 1775; écrit par Jacques Cook, commandant de la Résolution; dans lequel on a inséré la relation du Capitaine Furneaux, \& celle de Forster. Enrichi de plans, cartes, planches, portraits et vues par Hodges.

Duclos-Guyot, Nicolas-P. Journal... ? (Capitán de la Boudese en el viaje de Bougainville).

Feuillée, Louis. 1714. Journal des observations physiques; mathématiques et botaniques faites par I'ordre du Roy sur les côtes Orientales de l'Ámérique M éridionale... Paris, 3 vols.

Frezier, Amedée François. 1716. Relation du Voyage dela M er du Sud aux Côtes du Chili et du Pérou, fait pendant les années 1712, 1713 \& 1714. Paris.

Giraudais, François Chenard de la (capitán de l'Étoile con Bougainville).

Hawkesworth, John. 1774. Relation des voyages entrepris par ordre de sa M ajesté Britanique pour faire des découvertes dans I'hémisphère meridional, et succesivement exécutés par Byron, Carteret, Wallis et Cook. Paris.

- 1774. Relations des voyages entrepris par ordre de sa majesté britannique, et successivement exécutés par leCommodore Byron, leCapitaine Carteret, le Capitaine Wallis \& le Capitaine Cook, dans les Vaisseaux le 
Dauphine, leSwallow \& I'Endeavour (l'édition anglaise de 1773 ajoute: drawn up from the papers of Banks).P, Saillant et Nyon; Panckoucke 1774, 8 volumes en-8 ${ }^{\circ}$ de LXXX-327, 347, 447, 462, 374, 312, 336 et 336 pp. De la misma fecha una edición en $4^{\circ}$ en 4 volumes avec 52 planches. 500 / 750.

Kolbe, Peter 1743. Description du Cap de Bonne-Espeance; ou I'on trouve tout ce qui concernel'histoire naturelle du pays; la religion, les mœaurs \& les usages des H otentots; et l'ètablissement des H olandais. Amsterdam, Jean Catuffe, 3 vols ( $3^{\text {rd }}$. edition).

Linnaei, Caroli. 1758. Systema Naturaeper regna tria naturae, secundum Classes, Ordines, Genera, Species... Edición 10, Tomo 1, Holmiae.

_ . 1766-1767. Systema Naturae per regna tria naturae... Edición 12 reformata, Tomo 1, Holmiae.

Pando, Felipe Abate [jesuita chileno en destierro junto con F. Gómez de Vidaurre y J.I. Molina; citado sólo por su nombre].

Pauw, Cornelio de, 1768-69. Recherches philosophiques sur les américaines... Berlin.

Pernetty, Dom 1770, 1773. Histoire d'un voyage aux îles M alouines en 1763-64 avec des observations sur le detroit de M agellan et sur les Patagons. Paris, 1 vol. $4^{\circ}$ (1770), 2 vols., $8^{\circ}$ (1773).

Robertson, William 1777. The history of America. London.

Spilbergen, Joris van, 1619. Speculum orientales occidentalisque Indiae navigationum quórum una... classis cum potestate Praefecti, altera Iacobi le Maire... Annis 1614, 15, 16, 17, 18. Lugduni Batauorum.

Ulloa, Antonio de y Juan Jorge y Ulloa, 1748. Relación histórica del viaje a la Amërica meridional hecho para medir algunos grados de meridiano terrestrey venir por ellos en conocimiento de la verdadera figura y magnitud de la tie rra..., Madrid, 4 vols. Traducida al alemán en 1753, al francés en 1752 y al inglés en 1758.

Wallis, Samuel. An account of a voyage round the world, in the years 1766,1767, and 1768 (Véase Hackesworth, 1777).

Viajeros... (cita de Molina sin detalles. Referencias posibles de su época).

. Histoire générale des voyages ou nouvelle collection de toutes les relations des voyages par mer et par terre, qui ont été publiées jusqu'à présent dans les differénts langues, etc. Paris, 1756-1759, 15 vols. en $4^{\circ}$.

- H istoria general de los viages ó nueva colección de todas las relaciones de los que seha hecho por mar y tierra y sehan publicado hasta ahora en diferentes lenguas de todas las naciones conocidas, etc. Obra traducida del inglés al francés por el Abate Antonio Francisco Prevost y al castellano por don Miguel Terracita. Aumentada con la relación de los últimos viajes que se ha hecho en este siglo. Madrid, 1763-1788, 26 volúmenes en $4^{\circ}$.

En esta cita de Feliz Cruz (1966, vol. 2: 299) agrega: ... en el tomo XVII: Viages de Magallanes, Noort etc.; XVIII, Drake, Sarmiento, Narborough, Genes, Rogers, Frezier, Anson; XIX, Anson, Dampier, Schouten, Pizarro, descubrimiento de Chile por Diego de Almagro etc.; XXIII, Jorge Juan, Frezier, descripción de Santiago, etc. (Nota de J.T. Medina).

. H istoire des navigations aux terres australes, contenant cequel'on sait des 
moeurs, et des productions, des contrées découvertes jusqu'à ce tour, et oû il est traitédel' utilitéd' y faire des plus amples découverteset des moyens d' y former un établissement ( par le Président des Brosses). Paris, 1756, 2 vols.

. Voyages dans la M er du Sud par les espagnoles et les hollandais. Paris, 1774, 1 vol., mapas. 